\title{
Multi-objective optimized genomic breeding strategies for sustainable food improvement
}

\author{
Deniz Akdemir ${ }^{1} \cdot$ William Beavis ${ }^{2} \cdot$ Roberto Fritsche-Neto $\mathbb{D}^{3} \cdot$ Asheesh K. Singh $\mathbb{(}^{2} \cdot$ Julio Isidro-Sánchez $\mathbb{(}^{4}$
}

Received: 27 February 2018 / Revised: 9 August 2018 / Accepted: 22 August 2018 / Published online: 27 September 2018

(c) The Author(s) 2018. This article is published with open access

\begin{abstract}
The purpose of breeding programs is to obtain sustainable gains in multiple traits while controlling the loss of genetic variation. The decisions at each breeding cycle involve multiple, usually competing, objectives; these complex decisions can be supported by the insights that are gained by applying multi-objective optimization principles to breeding. The discussion in this manuscript includes the definition of several multi-objective optimized breeding approaches within the phenotypic or genomic breeding frameworks and the comparison of these approaches with the standard multi-trait breeding schemes such as tandem selection, independent culling and index selection. Proposed methods are demonstrated with two empirical data sets and simulations. In addition, we have described several graphical tools that can aid breeders in arriving at a compromise decision. The results show that the proposed methodology is a viable approach to answer several real breeding problems. In simulations, the newly proposed methods resulted in gains larger than the methods previously proposed including index selection: Compared to the best alternative breeding strategy, the gains from multi-objective optimized parental proportions approaches were about 20-30\% higher at the end of long-term simulations of breeding cycles. In addition, the flexibility of the multi-objective optimized breeding strategies were displayed with methods and examples covering non-dominated selection, assignment of optimal parental proportions, using genomewide marker effects in producing optimal mating designs, and finally in selection of training populations for genomic prediction.
\end{abstract}

\section{Introduction}

There are two ways in which the action of a breeder can change the genetic properties of the population; the first by the choice of individuals to be used as parents, which constitutes selection (Allard 1999; Falconer et al. 1996) and the second by control of the way in which the parents are

Electronic supplementary material The online version of this article (https://doi.org/10.1038/s41437-018-0147-1) contains supplementary material, which is available to authorized users.

Deniz Akdemir

akdemir@cornell.edu

1 Cornell Statistical Consulting Unit, Cornell University, Ithaca, NY, USA

2 Department of Agronomy, Iowa State University, Ames, IA, USA

3 Department of Genetics, "Luiz de Queiroz" Agriculture College, University of Sao Paulo, Sao Paulo, Brazil

4 Agriculture \& Food Science, Animal and Crop section, University College Dublin, Dublin, Ireland mated, which embraces inbreeding and cross-breeding (Akdemir and Sánchez 2016; Fernández et al. 2001; Kinghorn and Shepherd 1999; Pryce et al. 2012; Shepherd and Kinghorn 1998; Sun et al. 2013; Wright 1921). Selection means breeding from the "best" individuals whatever "best" might be (Allard 1999). The simplest form of selection is to choose individuals based on their own phenotypic values. Nevertheless, the breeding value (BV) of an individual is what influences the next generation. If breeders choose individuals to be parents according to their phenotypic values, their success in changing the population can be predicted only from knowledge of the degree of correspondence between phenotypic values and BVs (heritability) (Cockerham 1963; Dudley and Moll 1969; Holland et al. 2003).

Breeders have been selecting on the basis of phenotypic values since domestication of plants and animals. More recently, breeders have substantially used the pedigreebased prediction of genetic values for the genetic improvement of complex traits (Crossa et al. 2006; Gianola and Fernando 1986; Henderson 1984; Piepho et al. 2008). 
The enhancements in high throughput genotyping (Lander et al. 2001; Margulies et al. 2005; Metzker 2010) have transformed breeding pipelines through markerassisted selection (MAS) (Lande and Thompson 1990), marker-assisted introgression (Charcosset and Hospital 1997), marker-assisted recurrent selection (Bernardo and Charcosset 2006), and genomic selection (GS) (Meuwissen et al. 2001). The latter uses genome-wide markers to estimate the effects of all genes or chromosome positions simultaneously to calculate genomic estimated breeding values (GEBVs), which are used for the selection of individuals. This process involves the use of phenotypic and genotypic data to build prediction models that would be used to estimate GEBVs from genome wide marker data. It has been proposed that GS increases the genetic gains by reducing the generation intervals and also by increasing the accuracy of estimated BVs.

The economic value of the final product in a breeding program generally depends on more than one trait (Bernardo 2002; Lynch et al. 1998). Hence, determining which individuals to select to be the parents of the next generation forces the breeder to consider several different characteristics.

This is usually referred to as multiple-trait selection and implied selection for correlated traits. Not all the correlated traits are equally important or all independent of each other, but they are of interest for two main reasons in breeding programs.

Firstly, to understand the genetic causes of correlation through the pleiotropic action of genes or physical linkage of genes. Secondly, because it is key to understand how the improvement of one trait will trigger concurrent changes in other traits (Allard 1999).

There are many ways of selecting for multiple traits but these will not often be equally efficient. The most efficient method is that which results in the maximum genetic improvement per unit of time and effort expended (Hazel and Lush 1942; Smith 1936).

One might select each trait singly in successive generations (tandem selection) until each trait is improved to a desired level. Tandem selection is practical when some traits can be meaningfully evaluated in the earlier stages of a breeding program and other traits can be evaluated only later (Acquaah 2009; Burgess and West 1993; Hallauer and Miranda 1987). One might select for all the traits at the same time but independently, rejecting all individuals that fail to come up to a certain standard for each trait regardless of their values for any other of the traits (independent culling levels) (Hazel 1943). Only individuals that meet the minimum or maximum standards for each trait are selected.

Most breeders have to deal with selection of multiple traits simultaneously (in pure lines, inbred lines, hybrids, clones, and synthetics). Therefore, multi-trait selection using a selection index is an attractive approach (Hazel and Lush 1942; Hazel 1943; Williams 1962). In index selection, the component traits are combined into a score or index, in such a way that selection applied to the index, as if the index were a single trait, will yield the most rapid possible improvement of economic value.

If all traits were collected easily and at the same time, the index selection could be applied within phenotypic selection (PS). When some trait values are missing, GEBVs obtained by genomic prediction can be the basis for index calculations. A major challenge that remains in index selection is the apriori weight assignment of economic values for different traits. Each breeding program has potentials for genomic improvement in the traits of interests defined by the genetic composition of the breeding population which might make certain breeding goals more easily attainable for that breeding program compared to others. Pre-assigned economic weights do not necessarily represent specific potentials of a breeding program. A parent breeding population's potentials can be defined as the lengths of all the paths between the mean BV of the individuals in this breeding population and the mean $\mathrm{BV}$ in a progeny breeding population that lead all the traits in the desired direction. If we assume that the gains in these traits have equal importance, then preferring longest path progeny population would mean we would be accumulating as many beneficial allele effects as possible in one cycle of breeding. The potentials concept can be extended to more than one generations, by calculating paths between the parent and progeny populations obtained in more than one cycle.

Index selection will not necessarily include the best individuals with respect to individual traits (for example see Supplementary Fig. 8. This can be seen as a drawback of the method because not including the best individuals for individual traits can lead to loss of beneficial alleles. In addition, index selection method does not control for inbreeding.

The multitrait breeding problem pose a fundamental question in terms of the best procedure to reach the breeding goals. In the last years, the great innovation in computer science have allowed to test new statistical methods to model uncertainty in multi-trait selection not just to improve selection (Bernardo and Yu 2007; Goddard 2009; Heffner et al. 2009; Meuwissen et al. 2001) but also as a tool to facilitate ideotype design in crop modelling (Casadebaig et al. 2016; Gouache et al. 2017; Martre et al. 2015; Picheny et al. 2017). Numerical models can predict the outcome of plant traits by simulating physiological processes and their interaction with the environment (Ghanem et al. 2015; Martre et al. 2015; Rötter et al. 2015).

In this article, we propose an approach to multi-trait breeding based on a multi-optimization framework by setting optimal compromise solutions (Pareto front) that 
should be identified by an effective and complete search procedure to let the breeder to carry out the best choice. A novelty of our approach is that it extends some of the previously proposed breeding approaches, such as optimal parental contributions to multiple traits. However, the MOOB framework provides a framework with which many other breeding problems can be answered. For instance, we have included an illustration of MOOB framework for selection of training populations in the discussions section.

This article describes methods for sustainable improvement of crops and animals in rapidly changing environments by modeling genetic variability, optimizing breeding choices that involve trade-offs across multiple-traits, while controlling for the negative impacts of excessively high selection intensities on useful genetic variability.

The aim objectives of this article are (i) introduce the multi-objective optimization framework for plant and animal breeding and (ii) to compare the efficiency of the methods of this framework with previous multiple trait approaches.

\section{Materials}

\section{Wheat and barley datasets}

The genetic material used in this study consists of two different datasets on wheat and barley. Both of these data sets were downloaded from the triticeae toolbox (https:// triticeaetoolbox.org) and more details about these datasets are provided in Table 1 . We will use a wheat and a barley data to illustrate the use of multi-objective optimized breeding (MOOB) approaches.

Table 1 Germplasm description summary and heritability values for each trait of Wheat and Barley datasets

\begin{tabular}{lll}
\hline & Wheat data & Barley \\
\hline Individuals & 250 & 300 \\
Markers & 22,620 & 4419 \\
Environments & 2 & 4 \\
Traits & 2 & 3 \\
Heritability & & \\
Yield & 0.65 & 0.32 \\
Grain protein content (GPC) & 0.78 & 0.28 \\
Height & - & 0.44 \\
Genetic correlation & & \\
Yield-GPC & -0.3 & -0.27 \\
Yield-height & - & 0.55 \\
Height-GPC & - & -0.14 \\
\hline
\end{tabular}

\section{Model for estimating BVs}

For each dataset, the GEBVs for the traits grain yield (GY) and grain protein content (GPC) were predicted from a multi-trait mixed model with environment as fixed effects and $\mathrm{BVs}$ of individuals as random effects having a zero centered matrix-variate normal distribution with a separable covariance structure (Akdemir and Gupta 2011; Henderson and Quaas 1976; Montesinos-López et al. 2016) for traits and genotypes (Supplementary Equation 1). A similar model was assumed for the random residual terms. The GEBVs obtained from the above model were centered and standardized to bring the GEBVs to the same scale.

\section{Simulations}

The long-term performances of multi-trait breeding methods were evaluated by simulations. Beginning with two distinct founder genotypes, we have formed a population of $N(N=$ 100, 200, 300, 400) genotypes with 1000 single nucleotide polymorphism (SNPs) at three chromosomes each; and carried this population through 100 generations of random mating. Two traits were defined simultaneously by attaching random quantitative trait loci (QTL) effects at 200 randomly selected loci on each chromosome where 100 of these were taken to have opposite sign effects for the different traits, using these effects to calculate the genotypic values for each individual and adding to each of these a value generated from a normal distribution with zero mean and variance equal to the variance of the genotypic values in the population. The traits in this initial population were negatively correlated and each had a heritability value of 0.5 . Heritability of the traits were kept constant through the cycles of the simulations. The base population for breeding simulations were obtained by simulating 10 rounds of tandem selection over two traits with $50 \%$ selection intensity. Thirty replications of 16 cycles GS and 10 cycles of PS with tandem, index selection and culling, and 16 cycles with three multi-trait breeding methods recommended in the manuscript have been simulated starting from this base population. Marker effects were estimated from simulated phenotypic and genotypic data on the current population data at odd numbered breeding cycles.

\section{Methods}

In this section, we will describe two new approaches along with the standard methods for multi-trait breeding. We will illustrate and compare these methods with empirical data sets and with simulation studies. More details about the multi-objective optimization techniques can be found in 
Coello (2006), Deb (2001), Konak et al. (2006) and references there in.

\section{Multi-objective optimization and related concepts}

A single-objective optimization problem is defined as the minimization (or maximization) of a scalar objective function $f(\boldsymbol{x})$ subject to inequality constraints $g_{i}(\boldsymbol{x}) \leq 0, i=1, \ldots$, $m$ and equality constraints $h_{j}(\boldsymbol{x})=0, j=1, \ldots, p$, where $\boldsymbol{x}$ is a $n$-dimensional decision variable vector.

Multi-objective problems are those problems where the goal is to optimize simultaneously $k$ objective functions designated as: $f_{1}(\boldsymbol{x}), f_{2}(\boldsymbol{x}), \ldots, f_{k}(\boldsymbol{x})$ and forming a vector function $F(\boldsymbol{x})=\left[f_{\ell}(\boldsymbol{x})\right]_{\ell=1}^{k}$ subject to inequality constraints $g_{i}(\boldsymbol{x}) \leq 0, i=1, \ldots, m$ and equality constraints $h_{j}(\boldsymbol{x})=0, j=$ $1, \ldots, p$.

Although single-objective optimization problems may have a unique optimal solution, multi-objective optimization problems (as a rule) present a multiplicity of compromise solutions, i.e., Pareto optimal solutions are those solutions within the decision space whose corresponding variables cannot be all simultaneously improved.

Non-dominated ordering implements the concept of dominance (not to be confused with the dominance concept in genetics that describes the relationship between alleles of one gene) and classifies a population of solutions into boundaries according to their level of dominance. The first level includes all the non-dominated solutions, the second level are formed by the non-dominated solutions after excluding the solutions in the first level and this allocation process finishes when all solutions are allocated within their respective frontiers. After this process, the first-frontier solutions are not dominated by any other individual; however, they dominate the second frontier. Thus, solutions of the $i$ th frontier dominate individuals of the $(i+1)$ th frontier, i.e, solutions can be sorted according to these frontiers (see Supplementary Fig. 1). Each circle in Fig. 2a, b is a genotype, and dominance ordering connects all genotypes of the same dominance with a line. The genotypes on the lower dominance ordering levels are preferable to genotypes in the higher ordering levels and these genotypes with lower dominance ordering levels should be assigned higher weights in selection.

Non-dominance ordering and assignment of parental contribution proportions based on the above ideas are demonstrated with an hypothetical example in Supplementary Fig. 1a-f.

\section{Selecting a "good" solution on the frontier surface}

At the end of a multi-objective optimization, the decision maker (DM) has to select the preferred solutions from the Pareto frontier; this can be a difficult task for high- dimensional multi-objective optimization problems. For this reason, decision-making support tools are developed to aid the DM in selecting the preferred solutions. The choice of a unique solution in the collection of Pareto optimal solutions depends on the knowledge of problem characteristics, and a solution in a particular model may not be the best in another model or environment. For two-dimensional and threedimensional multi-objective optimization problems a strategy is to first plot the Pareto frontier followed by visual identification of the kink (knee) of the frontier as the region of preferred solutions. Some methods of finding knees in multi-objective optimization are described in Branke et al. (2004). An heuristic approach for identifying preferred solutions on the frontier can be defined by using the ideal solution concept and global criterion (see Supplementary Information). Some other decision support tools for multiple-criterion decision-making were described in Agrawal et al. (2005), Zio and Bazzo (2012), and Tušar and Filipič (2015).

\section{Self-organizing maps (SOMs) for visualizing the Pareto optimal solutions}

SOMs (Kohonen 1981, 1998) have been recommended for visualizing the Pareto optimal solutions for highdimensional multi-objective problems (Obayashi and Sasaki 2003). Neural networks are used in learning tasks that are too complex for human brain to comprehend and $\mathrm{SOM}$ is a unsupervised neural networks technique for organizing complex or vast amounts of data by providing lower dimensional representations of data in manner that is most easily understood. Specifically, SOMs are a type of artificial neural network that provides a topology preserving mapping from the high-dimensional space to map units. The property of topology preserving means that the mapping preserves the relative distance between the points; points that are near each other in the input space are mapped to nearby map units in the SOM. The SOM can thus serve as a cluster analyzing tool of high-dimensional data and be used as a visual aid in determining a 'good' solution on the frontier surface. We have provided two examples that illustrate the use of SOMs in the context of MOOB in Supplementary Figs. 13 and 14.

\section{MOOB strategies}

GS is being used increasingly in plant breeding to accelerate genetic gain (Crossa et al. 2010; Edriss et al. 2017; Gaynor et al. 2017; Roorkiwal et al. 2016). GS focuses on best performance of parents before mating, while genomic mating (GM) (Akdemir and Sánchez 2016) includes information on complementation of parents to be mated and thereby is more sustainable in the longer term. 
The standard breeding approaches, such as PS, GS, GM, and pedigree-based prediction, can be used with any of these multi-trait breeding approaches.

In the remaining of this article, we assume that a high density marker data is available for the current breeding population from which the co-ancestry coefficients can be calculated, and that there is no pedigree information. The implementation of PS in our simulations did not use any genotypic information or pedigrees. Basically, it referred to selecting the individuals with best observed phenotypes to be parents in the next generation.

\section{Non-dominated selection}

One approach involves sorting the individuals in a breeding program according to non-dominance ordering using the (predicted) BVs over the traits of interest. The assignments of parental contributions are done by assigning higher weights to individuals at lower non-dominance order.

Non-dominance ordering and assignment of parental contribution proportions based on the above ideas are demonstrated with an hypothetical example in Supplementary Fig. 1a-f.

\section{Multi-objective optimized genetic gains while controlling co-ancestry}

Parental contributions in the context of MOOB Let $A$ be the additive genetic relationships between the individuals in the genetic pool (this matrix can be obtained from genomewide markers for the individuals) and let $c$ be the vector of proportional contributions of individuals to the next generation under a random mating scheme. The average inbreeding and co-ancestry for a choice of $c$ can be defined as $r=1 / 2 c^{\prime} A \boldsymbol{c}$. If $\boldsymbol{b}$ is the vector of GEBVs, i.e., the vector of BLUP estimated BVs of the candidates for selection, the expected gain is defined as $g(\boldsymbol{c})=\boldsymbol{c}^{\prime} \boldsymbol{b}$. Without loss of generality, assume that the breeders long-term goal is to increase the value of $g(\boldsymbol{c})$.

Several authors (Brisbane and Gibson 1995; Clark et al. 2013; Meuwissen 1997; Sonesson et al. 2012) have proposed minimizing the average inbreeding and coancestry while restricting the genetic gain. These approaches find the parental proportions obtained by solving the following optimization problem: minimize $r$ $(\boldsymbol{c})=1 / 2 \boldsymbol{c}^{\prime} A \boldsymbol{c}$ subject to $\boldsymbol{c}^{\prime} \boldsymbol{b}=\rho$, and $\boldsymbol{c}^{\prime} 1=\mathbf{1}^{\prime} \boldsymbol{c} \geq 0$, where $\rho$ is the desired level of gain. This problem is easily recognized as a quadratic optimization problem (QP). There are many efficient algorithms that solves QPs so there is in practice little difficulty in calculating the optimal solution for any particular data set. Recently, several allocation strategies were tested using QPs in Goddard (2009), Pryce et al. (2012), and Schierenbeck et al. (2011).

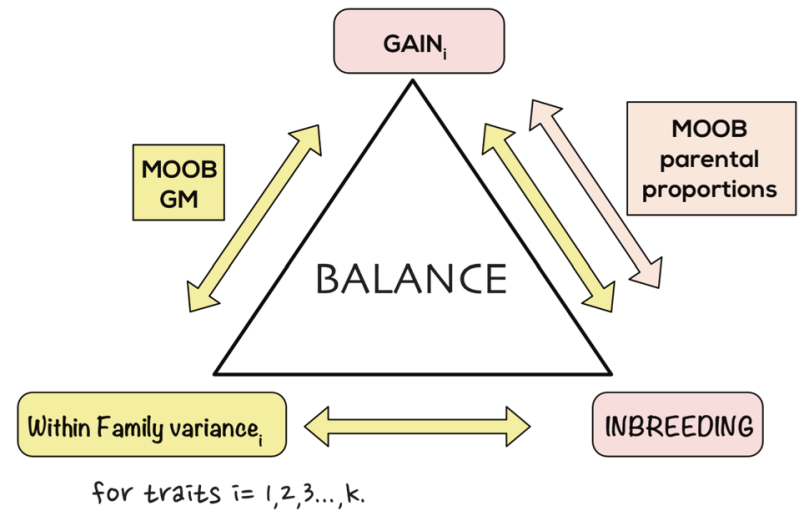

Fig. 1 An abstraction of methods for improving multiple traits under parental proportions and genomic mating. MOOB multi-objective optimized breeding. Assigning parental contributions involve balancing gains for $k$ traits and inbreeding. Genomic matings seeks to balance gains, within-family variances for $k$ traits and inbreeding

We suggest the following extension of the above formulation for obtaining parental proportions in the multi-trait scenario: Let $\boldsymbol{g}_{1}, \boldsymbol{g}_{2}, \ldots, \boldsymbol{g}_{k}$ denote the $m$-dimensional vectors of GEBVs for $k$ traits. We assume maximum is sought for each of these traits. As in Brisbane and Gibson (1995), Meuwissen (1997), Meuwissen et al. (2001), Wray and Goddard (1994) we want to keep inbreeding to minimum. This defines the multi-objective optimization problem (see Fig. 1); more formally we are looking to solve the maximization of the vector function

$F(\boldsymbol{c})=\left[f_{\ell}(\boldsymbol{c})\right]_{\ell=1}^{k+1}$,

with $f_{1}(\boldsymbol{c})=\boldsymbol{c}^{\prime} \boldsymbol{g}_{1}, f_{2}(\boldsymbol{c})=\boldsymbol{c}^{\prime} \boldsymbol{g}_{2}, \ldots, f_{k}(\boldsymbol{c})=\boldsymbol{c}^{\prime} \boldsymbol{g}_{k}, f_{k+1}(\boldsymbol{c})=$ $-\frac{1}{2} c^{\prime} A c$ subject to inequality constraints $c_{i} \geq 0, i=1, \ldots, m$ and equality constraint $\sum_{i=1}^{m} c_{i}-1=0$.

A slight modification of the above aims to penalize negative genetic correlations between trait pairs, and it involves changing $f_{k+1}(\boldsymbol{c})=-\frac{1}{2} \boldsymbol{c}^{\prime} A \boldsymbol{c}$ to

$f_{k+1}(\boldsymbol{c})=-\frac{1}{2} \boldsymbol{c}^{\prime}\left(\left(k-2 \sum_{i=1}^{k-1} \sum_{j>j}^{k} \Psi_{i, j}\right) A\right) \boldsymbol{c}$,

where $\Psi_{i, j}$ denotes the genetic correlation between traits $i$ and $j$. Both Eqs. (1) and (2) can be solved using QP.

The second class of multi-trait breeding schemes assigns parental proportions using one of the preferred solutions on the Pareto surface for the multi-objective problems stated in Eqs. (1) or (2).

\section{GM in the context of MOOB}

As opposed to the continuous parentage contribution proportions solutions in the GS method, the GM method gives the list of parent mates of the progeny (Akdemir and Sánchez 2016; Gorjanc and Hickey 2018; Kinghorn and 

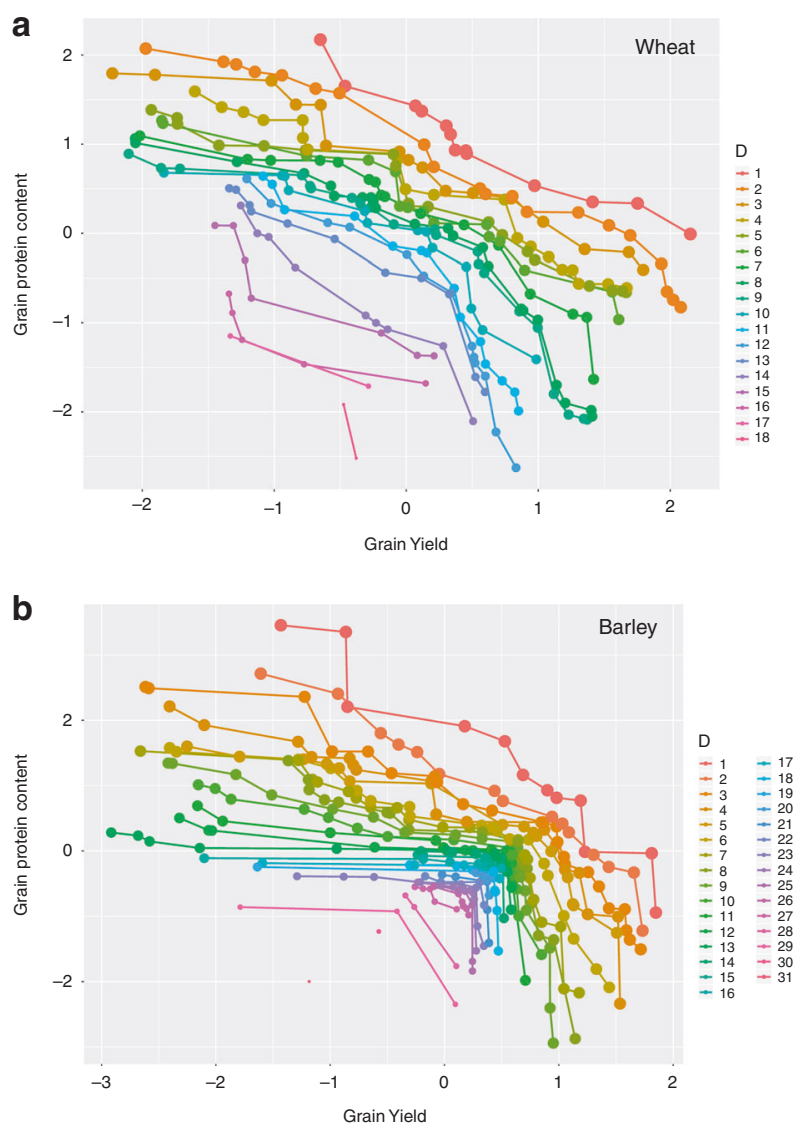

Fig. 2 These figures are obtained by plotting GEBVs from model in (eq:model) for grain yield and grain protein content in wheat (a) and barley (b) dataset. Each circle represents a genotype, and dominance ordering connects all genotypes of the same dominance with a line. The genotypes on the lower dominance ordering levels are preferable to genotypes in the higher ordering levels. Genotypes with lower dominance ordering levels should be assigned higher weights in selection. There are 18 and 31 levels of dominance for wheat and barley data sets. The axes in these figures measure the standardized GEBV values (i.e., centered by mean, scaled by standard deviation) for grain yield and grain protein content

Shepherd 1999; Kinghorn 2011). Multi-objective optimization problem (assuming maximization is sought for the trait) of the GM problem involves minimization of - Gain $(P),-$ Cross_Variance $(P)$ and Inbreeding $(P)$ with respect to mating plan $P$. Here, Gain $(P)$ represents the expected BVs, -Cross_Variance $(P)$ represents the expected variance in the BVs and Inbreeding $(P)$ measures the expected inbreeding of progenies obtained according to mating plan $P$.

Note that the measures of inbreeding in the optimal parental proportions and the GM approaches are different: The former is a measure of group co-ancestry while the latter is a measure of inbreeding of specific matings.

The expected gain for a mating plan can be calculated from the mid-parent genetic values. There are several alternative measures of inbreeding based on mating plans (Leutenegger et al. 2003; Wang 2011). In Akdemir and
Sánchez (2016), we have used a measure derived under the infinitesimal genetic effects assumption proposed by Quaas (1988) and Legarra et al. (2009). Measures of expected cross-variance (related to the risk measure in Akdemir and Sánchez (2016) and also to the usefulness concept in Jannink (2010)) can be obtained using the results in Akdemir and Sánchez (2016) under the assumption of unlinked markers. An alternative approach would be to use simulated progenies to calculate the cross-variances. One can also include information about the LD in these simulations. For example, Bernardo (2014) and Mohammadi et al. (2015), suggested to simulate progenies using the parental genotypes and a genetic map. Other measures of cross-variance were proposed in Lehermeier et al. (2017) and Zhong and Jannink (2007).

Extension to multi-trait GM for a $k$ trait problem (assuming maximization is sought for traits) is defined by the optimization problem which seeks minimization of $-\operatorname{Gain}(P)_{i},-$ Cross_Variance $(P)_{i}$ for $i=1:, 2, \ldots, k$ and Inbreeding $(P)$ with respect to mating plan $P$ (see Fig. 1).

Also note that the Results section below includes results obtained using the multi-objective optimized parental proportions approaches. The only instance of an example of the mating-based approach is reserved to the supplementary (Supplementary Figs. 13 and 14), where we display the frontier solutions for the GM-based approach. The readers can refer to the related R package "GenomicMating v2.0" (Akdemir et al. 2018) for an implementation of the multiobjective optimized GM designs.

\section{Results}

\section{Wheat and barley data sets}

Figure 2 and Supplementary Fig. 2 show the non-dominated orderings for wheat and barley datasets based on the GEBVs of traits GY and GPC.

The frontier surface related to the optimal parental proportions for the wheat and barley datasets is given in Fig. 3 and Supplementary Fig. 3. These figures show the frontier surfaces obtained by plotting Pareto optimal solutions for parental contributions obtained by solving the optimization problem given in Eq. (1) for improving GY and GPC while controlling group coancestry. A good solution can be visually detected by closely examining this surface to find a acceptable kink point, i.e., solutions of the Pareto-optimal front where a small improvement in one objective would lead to a large deterioration in at least one other objective. These solutions are sometimes also called "knees".

Figure 4 shows an example of two "good" solutions on the wheat frontier curve obtained from Fig. 3. Supplementary Fig. 4 as calculated by Eq. (2) also shows "good" 


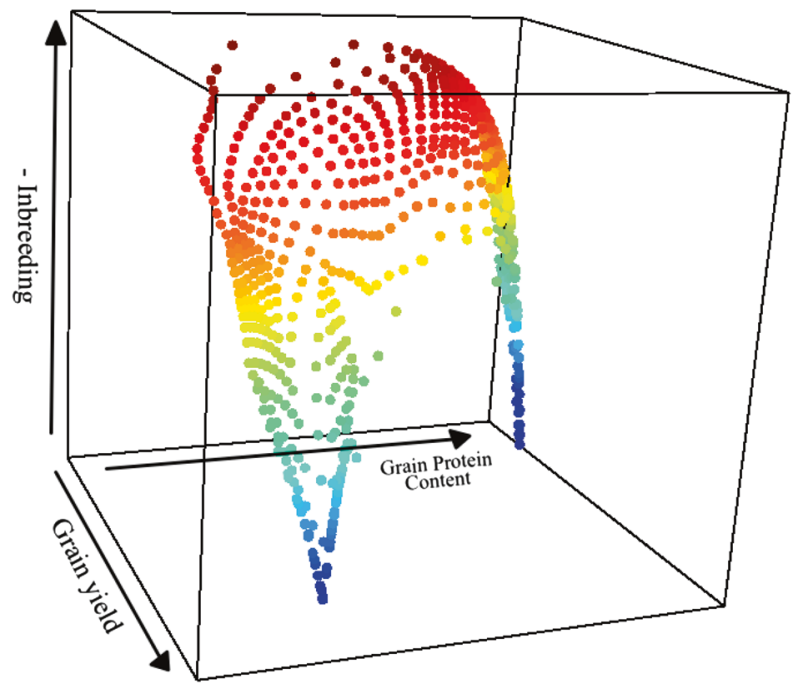

Fig. 3 Pareto optimal solutions for parental contributions (wheat data) obtained by solving the optimization problem giving in Eq. (1) for improving grain yield (GY) and grain protein content (GPC) while controlling group coancestry, i.e, we assume we want to maximize GY, GPC, and the negative of inbreeding. The redness of the points indicates closeness to ideal solutions as calculated by the formula in Supplementary Information Equation (Eq. (2))
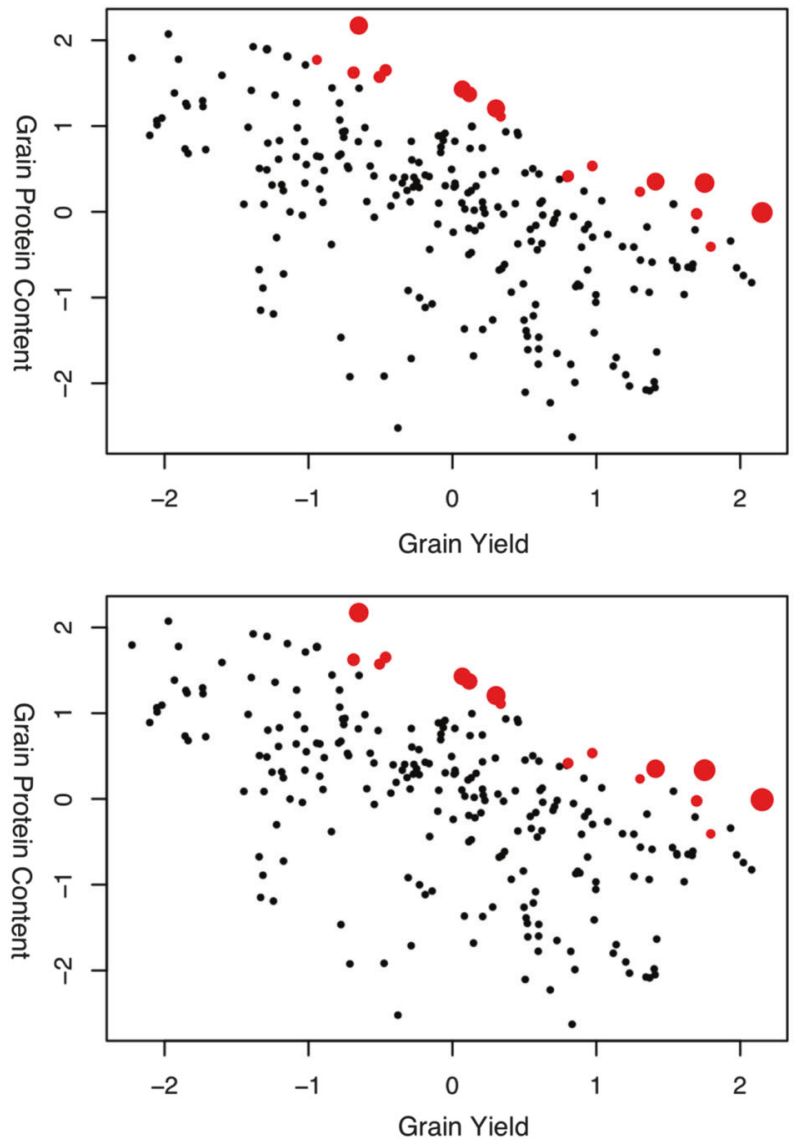

Fig. 4 Two 'good' solutions on the wheat frontier curve obtained from Fig. 3 for the wheat data. Red points indicated the individuals that have non-zero parental proportions. The size of the points are proportional solutions for the barley dataset. Note that non-dominated ordering-based approaches (Supplementary Fig. 2) give similar solutions to optimized parental proportions approach. Nevertheless, solutions for the parental proportions represent the control in inbreeding and have different weights for the individuals on the same level of dominance.

The multi-trait breeding described in this manuscript can also be used to improve one or more traits in several target environments. For example, a common breeding goal is improvement of yield in multiple environments. The nondominance ordering for the GEBVs for $G Y$ in the four barley dataset environments (dry-irrigated $\times$ high-low nitrogen) are given in Supplementary Fig. 7.

\section{Long-term performance evaluated by simulations}

Figure 5a-d shows the results from simulations for the study of the long-term behavior of PS and GS. Here, we use the standard methods of index and tandem selection, as well as, the independent culling method, along with the results for non-dominated selection, and the two forms of MOOB
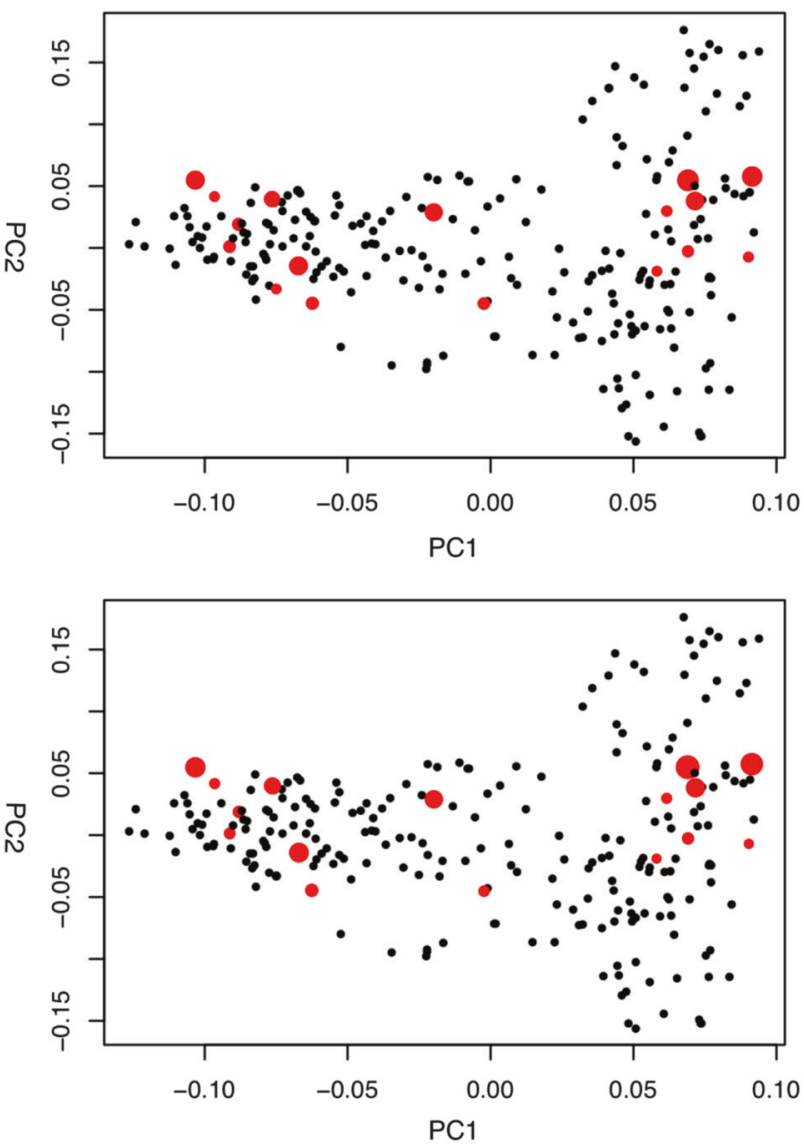

to the magnitude of the parental contributions. The figures on the right side, represent the same information but on the first two principal components of the genotyping marker space. PC principal components 

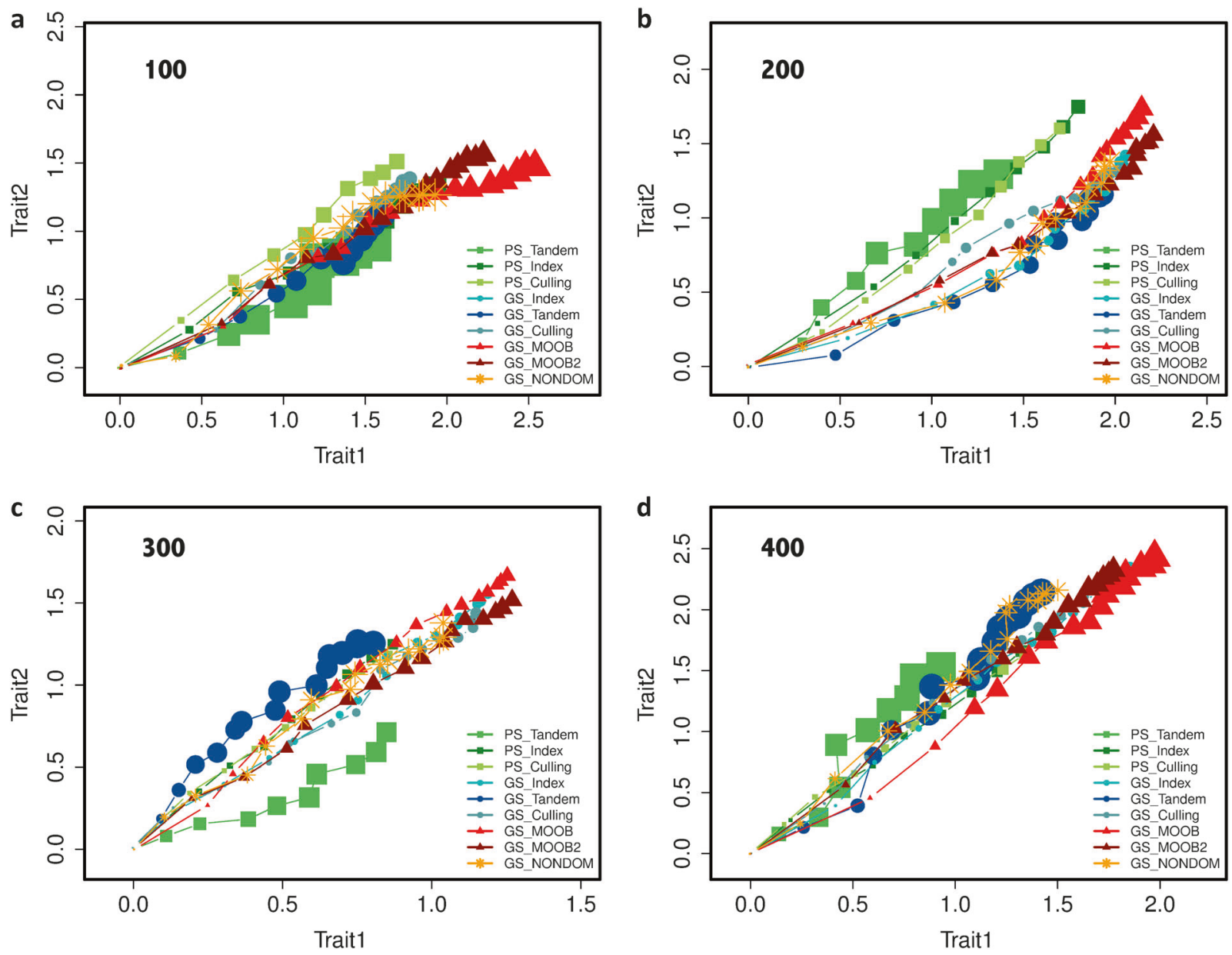

Fig. 5 Simulations: the results from 30 replications of 16 rounds GS and 10 rounds PS with tandem, index selection (equal weights for traits) and independent culling, and 16 rounds with three multi-trait breeding methods. Breeding population sizes 100, 200, 300, and 400. Data points (in the trend lines) represent the improvement in Trait 1 and Trait 2 in consecutive breeding cycles. The changing sizes of

schemes. The index weights for both traits were set to 0.5 in the simulations.

For the methods which involve genomic prediction, a multivariate mixed model was used to predict GEBVs of the traits at odd numbered cycles using the phenotypic values in that cycle as training data, which was then used in the next even numbered cycle to predict GEBVs of individuals in this cycle. The estimates of genetic covariance parameters were updated through the odd numbered cycles using the fitted model.

The simulation results indicate that the MOOB approach is more efficient (resulted in higher values in both Trait 1 and Trait 2 at the end of the breeding cycles) than classical methods. For all the population sizes, the MOOB approach performed more efficiently than any other methods by attaining the highest values of the BVs for both traits at the end of breeding cycles. Compared to the best alternative

points represents the variability of different breeding methods at each cycle over replicated trials of the experiment (30 reps) starting from the same initial population, the larger points corresponding to larger variances (the total variance of the mean breeding values obtained by a breeding method at a certain cycle calculated over the replications)

breeding strategy, the gains from multi-objective optimized parental proportions approaches were about 20-30\% higher at the end of long-term simulations of breeding cycles.

Selection of solutions on the frontier during these simulations were done using a weighted distance to the ideal solution (which was taken as the optimal values for the three statistics within the solutions on the frontier). The weights for populations of size 100 and 200 were fixed at $0.95,0.025$, and 0.025 corresponding to the measures of inbreeding, gain in Trait 1 and gain in Trait 2. We have increased the intensity of selection for populations of size 300 and 400 by changing these weights to $0.9,0.05$, and 0.05 . Note that, these values will be population specific and were fixed here only for the purposes of computer simulation of many cycles of breeding as described above. Decision support tools described previously should be utilized when applying the MOOB methods. 


\section{Discussion}

A major task of breeders is to increase the frequency of favorable alleles of quantitative traits, controlled by a large number of genes. After the choice of germplasm, a breeder uses some type of cyclical selection program to maximize the genetic improvement of desired traits. The important aspect is that superior materials selected for the breeding population should be recombined to obtain a new breeding population. Therefore, it is important to incorporate recurrent selection into classical breeding programs (Hallauer and Carena Filho 2010). One of the drawbacks of incorporating GS in breeding programs or even the traditional pedigree-based selection could potentially lead to greater rates of inbreeding than PS, especially when the accuracy of the method is low to moderate (Lin et al. 2017). Although, it has been shown that the inbreeding rate per generation of GS is less than pedigree selection (Daetwyler et al. 2007), GS could lead to higher inbreeding rates per year when compared to PS (Lin et al. 2017). A potential consequence of higher inbreeding is decreased survival, growth, and reproduction in outbreed plants (Lin et al. 2017). In addition, the response to selection per er cycle might be drastically reduced. Therefore, the accumulation of inbreeding from GS should be controlled to avoid those detrimental effects.

In this study, we have showed that optimization strategies that controls the loss of genetic diversity (MOOB framework) allows to (i) better conservate genetic variance in the breeding population, (ii) to better estimate of GEBVs and (iii) to have minor repercussions on the genetic gain (Eynard et al. 2017; Gorjanc et al. 2017).

In addition, we believe that the main promise of MOOB is that they provide the breeders with decision support tools that allow optimal exploitation of the breeding material. Breeders are routinely faced with the challenge to obtain an optimal decision with respect to multiple criteria. There is a clear need for planning tools to support effective decision making in this domain assisting the DMs in choosing an adequate strategy within the possibilities offered by the decision space.

Breeding through selection of superior genotypes for use as parents in future generations usually involves selection, which are based on more than one trait (Johnson et al. 1988). For example, although the GY is usually the primary trait of interest for most crops; plant height, earliness, stability, grain quality, stalk quality, abiotic and biotic stress tolerance, responsiveness, etc. (Mendonça et al. 2017, 2016) are also economically important traits. In assessing grain quality, elements of human subjectivity can be reduced by using multiple clearly defined traits observed by multiple raters to model grain quality (Stansell et al. 2017). Thus, simultaneous selection for several traits is necessary if recurrent selection methods are used because the selection that emphasizes only one trait can be detrimental to the overall agronomic performance of the germplasm (Hallauer and Carena Filho 2010). Consequently, the aim is to combine the favorable alleles that are present in different individuals, forming new and superior haplotypes.

As contrasted to the index selection methods, which require a prior decision on economic weights, the MOOB methodology involves selection of a 'good' solution among the optimal compromise solutions with the aid of decision support tools. The DM can come to a final decision by examining the frontier surface, or by using the ideal solution concept, by using the visualizations using SOMs. This addresses a major challenge in the application of index selection for many breeding programs: "how to assign weights?"

In our simulations, we have seen that MOOB outperforms other breeding approaches including the index selection. We would like to note that none of the compared approaches except MOOB controls for loss of genetic variation this is the main advantage of MOOB compared to the other methods. Nevertheless, it is possible also to imagine a scenario where the gains of and index is balanced with loss of genetic variation. This will lead to a muti-objective optimization problem which it is solved under MOOB framework.

We note that the multi-objective optimization methods can also be used in selection of training populations for genomic prediction. There has been many approaches that used a single selection criteria for designing genomic prediction training populations with the promise of improving genomic prediction accuracies and therefore improving expected gains from GS. Most of the literature is devoted to demonstrating the advantages and disadvantages of using one of these methods over the other (Akdemir et al. 2015; Isidro et al. 2015; Rincent et al. 2012). This debate can be somewhat circumvented by designing training populations that are optimal for multiple design criteria at the same time, in the multi-objective optimization framework. For example, a multi-objective optimized training population selection approach might seek solutions that balances genomic diversity in the training population, genetic closeness to a target population (the GS model trained in the training population will be used to predict GEBVs in the target population) in addition to some other criteria related to selection of training populations. This point is illustrated in Supplementary Fig. 15.

In this manuscript, we have used the multi-objective optimization approach in the context of multi-trait selection in breeding to efficiently select multiple traits simultaneously. Breeding for multiple traits is a real problem faced by breeders, usually because the choice of weightings in a selection index is inherently difficult due to the number of 
contributing factors. This paper presents a potential solution to this problem. In addition, MOOB is a flexible framework that can be used to answer many other problems in breeding, such as design of training populations. Future research directions therefore include finding other applications for MOOB related to breeding and more thoroughly evaluating the promises of this new framework in breeding.

Author contributions DA conceived the idea, analyzed the data, wrote a large part of the article, prepared the figures. JIS wrote a large part of the article, prepared figures. Authors BW, RFN, AKS contributed through discussions of the concepts and ideas and revision of the submitted manuscript.

\section{Compliance with ethical standards}

Conflict of interest The authors declare that they have no conflict of interest.

Open Access This article is licensed under a Creative Commons Attribution 4.0 International License, which permits use, sharing, adaptation, distribution and reproduction in any medium or format, as long as you give appropriate credit to the original author(s) and the source, provide a link to the Creative Commons license, and indicate if changes were made. The images or other third party material in this article are included in the article's Creative Commons license, unless indicated otherwise in a credit line to the material. If material is not included in the article's Creative Commons license and your intended use is not permitted by statutory regulation or exceeds the permitted use, you will need to obtain permission directly from the copyright holder. To view a copy of this license, visit http://creativecommons. org/licenses/by/4.0/.

\section{References}

Acquaah (2009) Principles of plant genetics and breeding. John Wiley \& Sons, Hoboken, NJ USA

Agrawal G, Bloebaum C, Lewis K (2005). Intuitive design selection using visualized $\mathrm{n}$-dimensional pareto frontier. In: 1st AIAA multidisciplinary design optimization specialist conference.

Akdemir D, Gupta AK (2011) Array variate random variables with multiway kro-necker delta covariance matrix structure J Algebraic Stat 2:98-113

Akdemir D, Sánchez JI (2016) Efficient breeding by genomic mating. Front Genet 7:210

Akdemir D, Sánchez JI, Haikka H, Brum IB (2018). GenomicMating: efficient breeding by genomic mating $\mathrm{R}$ package version 2.0.

Akdemir D, Sanchez JI, Jannink J-L (2015) Optimization of genomic selection training populations with a genetic algorithm. Genet Sel Evol 47:38

Allard (1999) Principles of plant genetics and breeding. John Wiley \& Sons, Hoboken, NJ USA

Bernardo R (2002) Breeding for quantitative traits in plants, vol 1. Stemma Press, Woodbury, MN USA

Bernardo R (2014) Genomewide selection of parental inbreds: classes of loci and virtual biparental populations. Crop Sci 54 (6):2586-2595

Bernardo R, Charcosset A (2006) Usefulness of gene information in marker-assisted recurrent selection: a simulation appraisal. Crop Sci 46(2):614-621

Bernardo R, Yu J (2007) Prospects for genomewide selection for quantitative traits in maize. Crop Sci 47(3):1082-1090
Branke et al (2004) Finding knees in multiobjective optimization. In International conference on parallel problem solving from nature. Springer, Berlin, Heidelberg Germany, pp 722-731

Brisbane J, Gibson J (1995) Balancing selection response and rate of inbreeding by including genetic relationships in selection decisions. Theor Appl Genet 91(3):421-431

Burgess JC, West D (1993) Selection for grain yield following selection for ear height in maize. Crop Sci 33(4):679-682

Casadebaig P, Mestries E, Debaeke P (2016) A model-based approach to assist variety evaluation in sunower crop. Eur $\mathrm{J}$ Agron 81:92-105

Charcosset A, Hospital F (1997) Marker-assisted introgression of quantitative trait loci. Genetics 147(3):1469-1485

Clark SA, Kinghorn BP, Hickey JM, van der Werf JH (2013) The effect of genomic information on optimal contribution selection in live stock breeding programs. Genet Sel Evol 45(1):1

Cockerham CC (1963) Estimation of genetic variances. Stat Genet Plant Breed 982:53-94

Coello CC (2006) Evolutionary multi-objective optimization: a historical view of the field. IEEE Comput Intell Mag 1(1):28-36

Crossa J, Burgueño J, Cornelius PL, McLaren G, Trethowan R, Krishnamachari A (2006) Modeling genotype $\times$ environment interaction using additive genetic covariances of relatives for predicting breeding values of wheat genotypes. Crop Sci 46 (4): $1722-1733$

Crossa J, de Los Campos G, Pérez P, Gianola D, Burgueño J, Araus JL, Makumbi D, Singh RP, Dreisigacker S, Yan J et al. (2010) Prediction of genetic values of quantitative traits in plant breeding using pedigree and molecular markers. Genetics 186(2):713-724

Daetwyler HD, Villanueva B, Bijma P, Woolliams JA (2007) Inbreeding in genome-wide selection. J Anim Breed Genet 124 (6):369-376

Deb K (2001) Multi-objective optimization using evolutionary algorithms, vol 16. John Wiley \& Sons, Hoboken, NJ USA

Dudley J, Moll R (1969) Interpretation and use of estimates of heritability and genetic variances in plant breeding. Crop Sci 9 (3):257-262

Edriss V, Gao Y, Zhang X, Jumbo MB, Makumbi D, Olsen MS, Crossa J, Packard KC, Jannink J-L (2017) Genomic prediction in a large african maize population. Crop Sci 57(5):2361-2371

Eynard S, Croiseau P, Laloë D, Fritz S, Calus M, Restoux G (2017) Which individuals to choose to update the reference population? Minimizing the loss of genetic diversity in animal genomic selection programs G3: Genes,Genomes, Genetics 8:113-121

Falconer DS, Mackay TF, Frankham R (1996) Introduction to quantitative genetics (4th edn). Trends Genet 12(7):280

Fernández J, Toro M, Caballero A (2001) Practical implementation of optimal management strategies in conservation programmes: a mate selection method. Anim Biodivers Conserv 24(2):17-24

Gaynor RC, Gorjanc G, Bentley AR, Ober ES, Howell P, Jackson R, Mackay IJ, Hickey JM (2017) A two-part strategy for using genomic selection to develop inbred lines. Crop Sci 57 (5):2372-2386

Ghanem ME, Marrou H, Sinclair TR (2015) Physiological phenotyping of plants for crop improvement. Trends Plant Sci 20 (3): 139-144

Gianola D, Fernando RL (1986) Bayesian methods in animal breeding theory. J Anim Sci 63(1):217-244

Goddard M (2009) Genomic selection: prediction of accuracy and maximisation of long term response. Genetics 136(2):245-257

Gorjanc, G, Gaynor, RC \& Hickey, JM Theor Appl Genet (2018) 131: 1953

Gorjanc G, Hickey JM (2018) Alphamate: a program for optimising selection, maintenance of diversity, and mate allocation in breeding programs. Bioinformatics. 1-4 
Gouache D, Bogard M, Pegard M, Thepot S, Garcia C, Hourcade D, Paux E, Oury F-X, Rousset M, Deswarte J-C et al. (2017) Bridging the gap between ideotype and genotype: challenges and prospects for modelling as exemplified by the case of adapting wheat (triticum aestivum 1.) phenology to climate change in france. Field Crops Res 202:108-121

Hallauer A, Carena Filho M, Carena M (2010) Breeding Plans. In: Quantitative Genetics in Maize Breeding. Handbook of Plant Breeding, vol 6. Springer, New York, NY

Hallauer AR, Miranda J (1987) Quantitative genetics in maize breeding. Iowa State University Press, Ames

Hazel L, Lush JL (1942) The efficiency of three methods of selection. J Hered 33(11):393-399

Hazel LN (1943) The genetic basis for constructing selection indexes. Genetics 28(6):476-490

Heffner EL, Sorrells ME, Jannink J-L (2009) Genomic selection for crop improvement. Crop Sci 49(1):1-12

Henderson (1984) Applications of Linear Models in Animal Breeding. 1984, Guelph University Press, Guelph, Canada

Henderson C, Quaas R (1976) Multiple trait evaluation using relatives' records. J Anim Sci 43(6):1188-1197

Holland JB, Nyquist WE, Cervantes-Martínez CT (2003) Estimating and interpreting heritability for plant breeding: an update. Plant Breed Rev 22:9-112

Isidro J, Jannink J-L, Akdemir D, Poland J, Heslot N, Sorrells ME (2015) Training set optimization under population structure in genomic selection. Theor Appl Genet 128(1):145-158

Jannink J-L (2010) Dynamics of long-term genomic selection. Genet Sel Evol 42(1):35

Johnson BE, Dauer JP, Gardner CO (1988) A model for determining weights of traits in simultaneous multitrait selection. Appl Math Modell 12(6):556-564

Kinghorn B, Shepherd R (1999) Mate selection for the tactical implementation of breeding programs. Association Advancement Animal Breeding. Genetics 13:130-133

Kinghorn BP (2011) An algorithm for efficient constrained mate selection. Genet Sel Evol 43(1):1

Kohonen T (1981) Automatic formation of topologicalmaps of patterns in a self-organizing system. In: Proceedings of 2SCIA, Scand. Conference on Image Analysis. p 214-220, Helsinki, Finland

Kohonen T (1998) The self-organizing map. Neurocomputing 21 (1): $1-6$

Konak A, Coit DW, Smith AE (2006) Multi-objective optimization using genetic algorithms: a tutorial. Reliab Eng Syst Saf 91 (9):992-1007

Lande R, Thompson R (1990) Efficiency of marker-assisted selection in the improvement of quantitative traits. Genetics 124 (3):743-756

Lander ES, Linton LM, Birren B, Nusbaum C, Zody MC, Baldwin J, Devon K, Dewar K, Doyle M, FitzHugh W et al. (2001) Initial sequencing and analysis of the human genome. Nature 409 (6822):860-921

Legarra A, Aguilar I, Misztal I (2009) A relationship matrix including full pedigree and genomic information. J Dairy Sci 92 (9):4656-4663

Lehermeier C, Teyssédre S, Schön C-C (2017) Genetic gain increases by applying the usefulness criterion with improved variance prediction in selection of crosses. Genetics 207(4):1651-1661

Leutenegger A-L, Prum B, Génin E, Verny C, Lemainque A, ClergetDarpoux F, Thompson EA (2003) Estimation of the inbreeding coefficient through use of genomic data. Am J Hum Genet 73 (3):516-523

Lin Z, Shi F, Hayes BJ, Daetwyler HD (2017) Mitigation of inbreeding while preserving genetic gain in genomic breeding programs for outbred plants. Theor Appl Genet 130(5):969-980
Lynch M, Walsh B et al. (1998) Genetics and analysis of quantitative traits, vol 1. Sinauer Sunderland, MA

Margulies M, Egholm M, Altman WE, Attiya S, Bader JS, Bemben LA, Berka J, Braverman MS, Chen Y-J, Chen Z et al. (2005) Genome sequencing in microfabricated high-density picolitre reactors. Nature 437(7057):376-380

Martre P, He J, Le Gouis J, Semenov MA (2015) In silico system analysis of physiological traits determining grain yield and protein concentration for wheat as inuenced by climate and crop management. J Exp Bot 66(12):3581-3598

Martre P, Quilot-Turion B, Luquet D, Memmah M-MO-S, Chenu K, Debaeke P (2015) Model-assisted phenotyping and ideotype design. In : Crop physiology: applications for genetic improvement and agronomy. Victor O. Sadras, Daniel Calderini (eds.). Oxford : Academic Press, 349-373

Mendonca LF, Fritsche N, Granato Í, Alves FC et al. (2017) Accuracy and simultaneous selection gains for $\mathrm{N}$-stress tolerance and $\mathrm{N}$-use efficiency in maize tropical lines Scientia Agricola 74:481-488

Mendonca LF, Fritsche N, Granato Í, Alves FC et al (2016) Accuracy and simultaneous selection gains for grain yield and earliness in tropical maize lines. Maydica 61(3)

Metzker ML (2010) Sequencing technologies-the next generation. Nat Rev Genet 11(1):31-46

Meuwissen T (1997) Maximizing the response of selection with a predefined rate of inbreeding. J Anim Sci 75(4):934-940

Meuwissen THE, Hayes BJ, Goddard ME (2001) Prediction of total genetic value using genome-wide dense marker maps. Genetics 157(4):1819-1829

Mohammadi M, Tiede T, Smith KP (2015) Popvar: a genome-wide procedure for predicting genetic variance and correlated response in biparental breeding populations Crop Science 55:2068-2077.

Montesinos-López OA, Montesinos-López A, Crossa J, Toledo F, Pérez-Hernández O, Eskridge KM, Rutkoski J (2016) A genomic bayesian multi-trait and multi-environment model G3: Genes, Genomes, Genetics 6:2725-2744. https://doi.org/10.1534/g3.116. 032359

Obayashi S, Sasaki D (2003) Visualization and data mining of pareto solutions using self-organizing map. In: EMO. Springer, Berlin, Heidelberg Germany, pp 796-809

Picheny V, Casadebaig P, Trépos R, Faivre R, Da Silva D, Vincourt P, Costes E (2017) Using numerical plant models and phenotypic correlation space to design achievable ideotypes Plant, Cell \&Environment 40:1926-1939. https://doi.org/10.1111/pce.13001

Piepho H, Möhring J, Melchinger A, Büchse A (2008) Blup for phenotypic selection in plant breeding and variety testing. Euphytica 161(1-2):209-228

Pryce J, Hayes B, Goddard M (2012) Novel strategies to minimizeprogeny inbreeding while maximizing genetic gain using genomic information. J Dairy Sci 95(1):377-388

Quaas R (1988) Additive genetic model with groups and relationships. J Dairy Sci 71(5):1338-1345

Rincent R, Laloë D, Nicolas S, Altmann T, Brunel D, Revilla P, Rodriguez VM, Moreno-Gonzalez J, Melchinger A, Bauer E et al. (2012) Maximizing the reliability of genomic selection by optimizing the calibration set of reference individuals: comparison of methods in two diverse groups of maize inbreds (Zea mays 1.). Genetics 192(2):715-728

Roorkiwal M, Rathore A, Das RR, Singh MK, Jain A, Srinivasan S, Gaur PM, Chellapilla B, Tripathi S, Li Y et al. (2016) Genome enabled prediction models for yield related traits in chickpea Front Plant Sci 7:1666

Rötter R, Tao F, Höhn J, Palosuo T (2015) Use of crop simulation modelling to aid ideotype design of future cereal cultivars. J Exp Bot 66(12):3463-3476 
Schierenbeck S, Pimentel E, Tietze M, Körte J, Reents R, Reinhardt F, Simianer H, König H (2011) Controlling inbreeding and maximizing genetic gain using semi-defnite programming with pedigree-based and genomic relationships $J$ Dairy Sci 94:6143-6152

Shepherd R, Kinghorn B (1998) A tactical approach to the design of crossbreeding programs. In Proceedings of the sixth world congress on genetics applied to livestock production, vol 25, Armidale, 11-16 January 1998, pp 431-438

Smith HF (1936) A discriminant function for plant selection. Ann Hum Genet 7(3):240-250

Sonesson AK, Woolliams JA, Meuwissen TH (2012) Genomic selection requires genomic control of inbreeding. Genet Sel Evol 44(1):1

Stansell Z, Björkman T, Branham S, Couillard D, Farnham MW (2017) Use of a quality trait index to increase the reliability of phenotypic evaluations in broccoli. HortScience 52(11):1490-1495

Sun C, VanRaden P, O'Connell J, Weigel K, Gianola D (2013) Matingprograms including genomic relationships and dominance effects. J Dairy Sci 96(12):8014-8023
Tušar T, Filipič B (2015) Visualization of pareto front approximations in evolutionary multiobjective optimization: a critical review and the prosection method. IEEE Trans Evol Comput 19(2):225-245

Wang J (2011) Coancestry: a program for simulating, estimating and analysing relatedness and inbreeding coefficients. Mol Ecol Resour 11(1):141-145

Williams J (1962) The evaluation of a selection index. Biometrics 18 (3):375-393

Wray N, Goddard M (1994) Moet breeding schemes for wool sheep 1. Design alternatives Anim Prod 59:71-86

Wright S (1921) Systems of mating. I. The biometric relations between parent and offspring. Genetics 6(2):111

Zhong S, Jannink J-L (2007) Using quantitative trait loci results to discriminate among crosses on the basis of their progeny mean and variance. Genetics 177(1):567-576

Zio E, Bazzo R (2012) A Comparison of Methods for Selecting Preferred Solutions in Multiobjective Decision Making. In: Kahraman C. (eds) Computational Intelligence Systems in Industrial Engineering. Atlantis Computational Intelligence Systems, vol 6 pp 23-43. Atlantis Press, Paris 